\title{
Diclofenac and the Risk of Rhabdomyolysis: Analysis of Publications and the WHO Global Pharmacovigilance Database
}

\author{
Mulugeta Russom $^{1}$ (D) Yodit Fitsum ${ }^{1}$ (D) $\cdot$ Abiel Abraham $^{2}$ (D) $\cdot$ Ruth L. Savage $^{3,4,5}$ (D)
}

Accepted: 28 February 2021 / Published online: 30 March 2021

(c) The Author(s) 2021

\begin{abstract}
Background Diclofenac, a nonsteroidal anti-inflammatory drug, is not a documented cause of rhabdomyolysis in the Summaries of Product Characteristics held by major regulators. There are, however, eight published single case reports that associate rhabdomyolysis with diclofenac.

Objective Triggered by a serious local case report, this study was conducted to evaluate the evidence for a causal association between diclofenac and rhabdomyolysis.

Patients and Methods A descriptive analysis of rhabdomyolysis associated with diclofenac was conducted by mining data from the WHO Global Database of Individual Case Safety Reports, VigiBase, and published case reports.

Results 70 eligible cases were retrieved from VigiBase. The median age was 56.5 years (range 1-90). Where reported precisely (26 reports), the median time to onset of rhabdomyolysis following administration of diclofenac was 3 days. In 20 cases, diclofenac was reported as a sole suspect and was solely administered in 14 of these. In 30 cases, rhabdomyolysis abated following withdrawal of diclofenac. Seven of these cases fulfilled the WHO-UMC case-causality assessment criteria for 'probable'. Diclofenac was probably an indirect cause in another five reports where rhabdomyolysis ensued from injectionsite necrosis. There were eight fatalities and intramuscular administration was over-represented in this group. In 27 patients taking lipid-lowering agents, the incidence of acute kidney injury with rhabdomyolysis was $62.9 \%$ compared with $37.1 \%$ for the whole cohort. Off-label use of diclofenac for minor or undiagnosed conditions was reported.

Conclusion Currently available data suggests a causal link between diclofenac and rhabdomyolysis either directly or indirectly.
\end{abstract}

\section{Introduction}

Diclofenac is a widely used non-selective non-steroidal antiinflammatory drug (NSAID). To our knowledge, diclofenac is not known to cause rhabdomyolysis. There were, however, eight single case reports published between 1996 and 2018

Mulugeta Russom

satiswt@gmail.com

1 Eritrean Pharmacovigilance Centre, National Medicines and Food Administration, Ministry of Health, Asmara, Eritrea

2 Akordat Hospital, Ministry of Health, Akordat, Eritrea

3 Uppsala Monitoring Centre, Uppsala, Sweden

4 Division of Health Sciences, New Zealand Pharmacovigilance Centre, University of Otago, Dunedin, New Zealand

5 Department of General Practice, University of Otago, Christchurch, New Zealand that associate rhabdomyolysis with diclofenac [1-8]. The summary of product characteristics (SmPC) of diclofenac, all dosage forms, approved by the Medicines and Healthcare Products Regulatory Authority of the United Kingdom [9] and the US Food and Drug Administration (FDA) [10] and safety reviews of diclofenac by the European Medicines Agency (EMA) [11] and Therapeutic Goods Administration of Australia (TGA) [12] do not mention rhabdomyolysis as an adverse effect.

Rhabdomyolysis is an extreme breakdown of skeletal muscle tissues characterized by muscle necrosis and the release of intracellular muscle constituents into the circulation. It is marked by elevated serum creatine kinase (CK), muscle pain and myoglobinuria and, in severe cases, could lead to extreme enzyme elevations, electrolyte imbalance and acute kidney injury in $10-50 \%$ of patients [13, 14]. Many conditions can lead to acquired rhabdomyolysis including trauma and crush syndrome, vascular ischaemia, toxins, infections and sepsis, metabolic disorders of salt and water, and a number of drugs [14]. 


\section{Key Points}

Diclofenac is not known to cause rhabdomyolysis; it has only previously been associated with single case reports.

Triggered by a serious case report, the causal relationship of diclofenac and rhabdomyolysis was assessed by analysing publications and the WHO global database of individual case safety reports.

The available evidence suggests a causal link between diclofenac and rhabdomyolysis that warrants attention from healthcare professionals and regulators.

In Eritrea, diclofenac sodium/potassium injection is widely used even without prescription for the management of acute febrile illnesses, rheumatoid arthritis, gouty arthritis and for moderate to severe pain of known or unknown causes. Recently, the Eritrean Pharmacovigilance Centre received a well-documented single case report of rhabdomyolysis in a young adult shortly after administration of diclofenac $75 \mathrm{mg} / 3 \mathrm{~mL}$ injection [intramuscular (IM)] prescribed for a febrile illness of unknown cause. This study was carried out to investigate the possibility of a causal link between diclofenac and rhabdomyolysis by mining data from the World Health Organization (WHO) Global Database for Individual Case Safety Reports (ICSRs), VigiBase [15] and literature evaluation.

\section{Patients and Methods}

\subsection{Study Design and Data Sources}

This was a descriptive analysis of diclofenac and risk of rhabdomyolysis reported to the WHO Global Database for ICSRs, VigiBase [15]. VigiBase is a databank developed and maintained by the Uppsala Monitoring Centre (UMC), Sweden. It is the world's largest pharmacovigilance database with around 21.5 million ICSRs (during data retrieval) submitted from member states since the establishment of the WHO Programme for International Drug Monitoring in 1968. There are currently 136 member states.

\subsection{Data Retrieval Approach}

Data mining in VigiBase was carried out on 27 February 2020 using the search criteria: 'diclofenac' as the drug substance and 'rhabdomyolysis' as a MedDRA reaction preferred term [16]. Retrieved data were exported to excel spreadsheets for further descriptive analysis. Using UMC's tool developed for data mining and analysis, VigiLyze, demographic variables, diclofenac administration details, indications, co-reported adverse reactions with rhabdomyolysis, co-suspect, and concomitant medicines and patients' medical histories where available were evaluated. Reaction outcomes, de-challenge and rechallenge information, and seriousness of the reactions were also retrieved. The information component (IC) for the diclofenac-rhabdomyolysis combination was also noted. The IC value is a measure of the disproportionality of a drug-adverse drug reaction (ADR) pair in the database [17]. A positive $\mathrm{IC}_{025}$ value (the lower border of the credible interval for the IC value $>0$ ) is "a traditional threshold which indicates that a drug-ADR pair is reported more often than expected based on all reports in the database", thus showing a statistical signal.

To exclude potential duplicate case reports, the first search was made by setting 'de-duplicate'. After automatic removal of potential duplicates, reports were then manually reviewed one-by-one and those that were found to be additional potential duplicates or irrelevant/invalid were removed from the assessment. When two or more reports were suspected to be duplicates of a single case report, the one with better completeness of information was retained for analysis whilst any additional information from the other reports was added before they were removed.

\subsection{Case Assessment}

Causality assessment of the retrieved case reports was undertaken to ascertain the likelihood that they represented rhabdomyolysis as a previously undocumented or insufficiently documented adverse reaction to diclofenac. The WHO-UMC system for standardized case causality assessment was applied for this purpose using the criteria for the causality categories 'certain', 'probable', 'possible', 'unlikely', 'unclassified' and 'unclassifiable' [18]. An effort was made to identify 'certain' or 'probable' cases for best evidence of a causal relationship. This was carried out by finding reports in which diclofenac was the sole suspect medicine, the patient recovered on discontinuation of diclofenac, and there were no clear alternative causes for rhabdomyolysis. In addition, cases with more than one suspect medicine but with recovery following discontinuation of only diclofenac were included. For a 'certain' assessment, recurrence on rechallenge with diclofenac was also required. All abstracted case reports from all available data sources were examined as a case series by applying the Bradford Hill guidelines for causal inference, modified for pharmacovigilance [19]. This causality assessment framework includes nine viewpoints for consideration-strength of association, 
temporality, consistency, specificity, dose-response relationship, biological plausibility, experimental evidence, analogy and coherence-each of which, if fulfilled, provide additional support for the ADR hypothesis. Furthermore, subgroup analysis was made on the following two scenarios: (1) Case reports in which diclofenac was given by IM injection, as fatal outcomes were more often associated with this administration route. (2) Case reports with lipid-lowering agents reported as co-suspect, concomitant or interacting because of possible confounding due to the propensity of these medicines to cause rhabdomyolysis or the potential for a pharmacodynamic interaction with diclofenac.

\subsection{Labelling and Literature Search}

To assess available documentation on a relationship between diclofenac and rhabdomyolysis, SmPCs of diclofenac approved by major regulatory authorities including the MHRA [9] and FDA [10] were reviewed. The online drug information databases such as Martindale: the Complete Drug Reference [20], Drugdex [21] and the SIDER side-effect resource [22] were also searched. Further, efforts were made to find relevant publications, including case reports, in Google Scholar, EMBASE and PubMed using the following search criteria in titles of articles: 'Diclofenac' AND 'rhabdomyolysis'. Further, secondary search of relevant articles cited in the already retrieved ones was also carried out.

\section{Results}

\subsection{Case Presentation}

Details of the case of rhabdomyolysis associated with diclofenac reported from Eritrea are presented in Tables 1 and 2. This 25-year-old male patient developed local tissue necrosis at the site of an intragluteal diclofenac injection and subsequently rhabdomyolysis. The clinical progression was complex and tissue damage at the injection site, infection and prolonged immobility may all have contributed to rhabdomyolysis.

\subsection{Results of Literature and Labelling Search}

The SmPCs of diclofenac, including that of the innovator company, approved by major regulatory authorities do not mention rhabdomyolysis as an adverse effect [9, 10, 23]. In other drug information sources, Martindale's adverse drug reaction checker [20] for diclofenac does not include rhabdomyolysis but states "there may be pain and, occasionally, tissue damage at the site of injection when diclofenac is given intramuscularly". Drugdex quotes one of the published case reports [21]. The innovator company of diclofenac, Novartis, advises healthcare professionals to strictly follow advice for administering diclofenac IM injections to avoid adverse events at the injection site, which may result in muscle weakness, paralysis, hypoaesthesia and injection-site

Table 1 Case presentation of rhabdomyolysis associated with diclofenac injection in a 25- year- old male patient

December (Dec) 2, 2018 Patient admitted to emergency ward of a hospital with sudden onset of right hip joint swelling associated with pain and passage of bloody urine for a duration of 2 two days following use of diclofenac intramuscular, gluteal, for acute febrile illness of unknown origin

Dec 3-6, 2018

Pain and swelling progressed to the whole right thigh, associated with skin redness, discoloration and blister formation

Dec 6, 2018

Vomiting, dizziness, generalized body weakness, sudden onset drowsiness, profuse sweating. Provisional diagnosis: acute tubular necrosis and cellulitis possibly secondary to diclofenac injection. Treatment: ceftriaxone, hydrocortisone, paracetamol, pethidine, intravenous fluids

Dec 7, 2018

Transferred to a national referral hospital. Investigations: anaemia, abnormal liver and renal function, leukocytosis, hypertriglyceridaemia (Table 2). Malaria and Helicobacter. pylori infection excluded. Intravenous cloxacillin and metronidazole commenced

Dec 7-12, 2018

Transfused with 3 units whole blood, antibiotics continued. Onset confusion, anxiety, decreased level of consciousness, decreased urine output, cardiac ejection murmur and dyspnoea

Dec 12, 2018

Transferred to Intensive Care Unit (ICU) with a working diagnosis of acute kidney injury and deep vein thrombosis (DVT)

Dec 12-27, 2018

Remained in ICU. Urine output and thigh circumference monitored. Gluteal area swelling became necrotic and oozed blood that required daily debridement. DVT ruled out. Treatment: flucloxacillin, furosemide, allopurinol, IV fluids, calcium gluconate, sodium bicarbonate, blood components. Investigations: persisting renal and liver dysfunction, increased uric acid; white cell, red cell and platelet counts, serum cholesterol, high-density lipoprotein and lowdensity lipoprotein all normal (Table 2). Dark brown urine observed (sometime after initial haematuria) and serum creatine kinase elevated to $3500 \mathrm{IU} / \mathrm{L}$. (normal range: 24-195). Urine myoglobin measurement was not available. Patient diagnosed with rhabdomyolysis and acute kidney injury probably secondary to diclofenac 


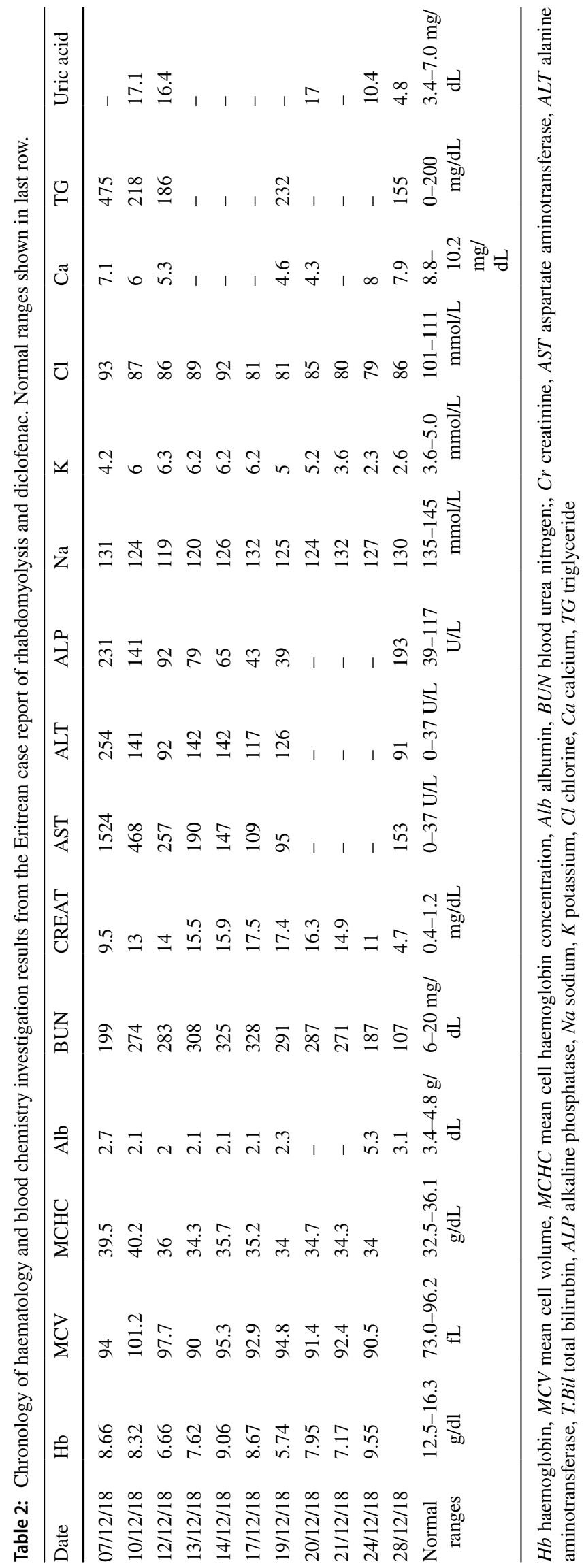


necrosis [23]. In an analysis of drug-induced rhabdomyolysis reported to the FDA, 100 cases were found to be associated with diclofenac but causality assessment was not undertaken in this study [24].

In the medical literature (Google Scholar, EMBASE and PubMed), there were no other published studies that associated diclofenac and rhabdomyolysis except the eight case reports [1-8]. We have assessed the causality of the published case reports, shown in Table 3, with the available evidence. In all these reports diclofenac was the sole suspect medicine and duration of its use to onset of rhabdomyolysis was consistently short at less than 10 days. Three of the published case reports of rhabdomyolysis fit the WHO-UMC criteria for 'probable' reactions $[1,5,6]$. In addition, two other patients died due to rhabdomyolysis in close temporal relationship to diclofenac administration with no apparent alternative explanations, one following an overdose [2] and one after developing necrotizing fasciitis at the injection site [7]. The cases reported by Delrio et al. [3], Knobloch et al. [4] and Schechner et al. [8] are classified as 'possible'. This is because the possibility that the patients were taking diclofenac for symptoms of rhabdomyolysis could not be excluded and in the case reported by Schechner et al. [8], a drug that is well known to cause rhabdomyolysis, cerivastatin, was concomitantly administered. This case report indicates how diclofenac may have increased the risk or severity of rhabdomyolysis with cerivastatin.

\subsection{Reports in the WHO Global Database of Individual Case Safety Reports, VigiBase}

In VigiBase, reports of rhabdomyolysis associated with diclofenac use first appeared in 1995. As of 27 February 2020, a total of 92 reports from 21 countries were identified and retrieved. Ten reports were removed by automated de-duplication. Manual case-by-case assessment revealed another 12 reports that were either invalid or potential duplicates, reducing the total reports for assessment to 70 (Fig. 1). Analysis of the 70 reports revealed that more than half were reported from 2012 onwards. They originated from 19 countries, mainly France (12), Germany (11), Japan (9), USA (7), and the UK (6). Over $60 \%$ of the reports were submitted by physicians. Age was given in 65 reports. The median was 56.5 years (range 1-90) and included two children. Approximately one-quarter $(27 \%)$ of the adult patients were aged less than 44 years. Sex was reported in all but two reports and the reaction manifested more in males (ratio 1.96:1.00).

Indications for diclofenac were listed in 40 reports. Ten patients were being treated for joint-related conditions and three for other specific musculoskeletal disorders. However, the majority of patients were being treated for non-specific, non-joint-related musculoskeletal pain or unspecified pain
(14) or a variety of other conditions including headache, fever, abdominal and post-operative pain (13).

Time to reaction onset (TTO), from the start of diclofenac treatment to onset of rhabdomyolysis, could be calculated precisely from 26 reports and approximately from a further six. From the 26 reports with precise dates, the median TTO was 3.0 days (range 1-87). For the six reports with less precise dates, TTO ranged from within 20 days to approximately 2 years. Overall, rhabdomyolysis onset was within 1 month of starting diclofenac in over 75\% (26/32) of the reports for which TTO could be calculated precisely or approximately. The route of administration was oral (31), IM (14), rectal (5), topical (3), intravenous (IV) (1) and unknown or other (16).

The reaction was marked as serious in 54 case reports and outcome was reported as 'recovered' (25) or 'recovered with sequelae' (3), 'recovering' (16), 'not yet recovered' (6), 'fatal' (7) or unknown (13). Acute kidney injury (37.1\%), increased CK, myalgia and metabolic acidosis were the most frequently co-reported reaction terms with rhabdomyolysis. Lipid-lowering agents were reported as co-suspects, interacting or concomitants in 27 reports.

In 20 case reports, diclofenac was reported as the sole suspected medicine, and in 14 of these 20 it was the sole administered medicine. Rhabdomyolysis was reported to have abated following withdrawal of diclofenac in ten of the 20 reports. The route of diclofenac administration in these reports was predominantly IM (11/20) in contrast with the whole dataset. Where diclofenac was not the sole suspect, lipid-lowering (LL) agents, paracetamol, furosemide and aspirin were the most frequently reported co-suspect medicines. In these case reports the route of diclofenac administration was predominantly oral.

Fatalities Overall, four males and three females died due to rhabdomyolysis. Two were children aged 2 years or less and five were adults with a median age of 39 years. Diclofenac was either the sole medicine or sole suspect medicine in four reports. The route of administration was IM (4), IV (1), rectal (1) and oral (1). Following IM injection, two patients developed a necrotizing soft tissue infection, one with sepsis (this patient had malignant cells in the bone marrow), and a third developed purpura fulminans. All are known complications of IM diclofenac injection and presumably led to rhabdomyolysis. The fourth IM injection was an overdose in a child who had $10 \%$ second-degree burns causing pain [2]. The patients who received rectal or oral administration, including the other child, had other exposures and conditions that were alternative explanations for rhabdomyolysis. The seventh patient was given diclofenac intravenously for thigh pain that was subsequently diagnosed as rhabdomyolysis. 


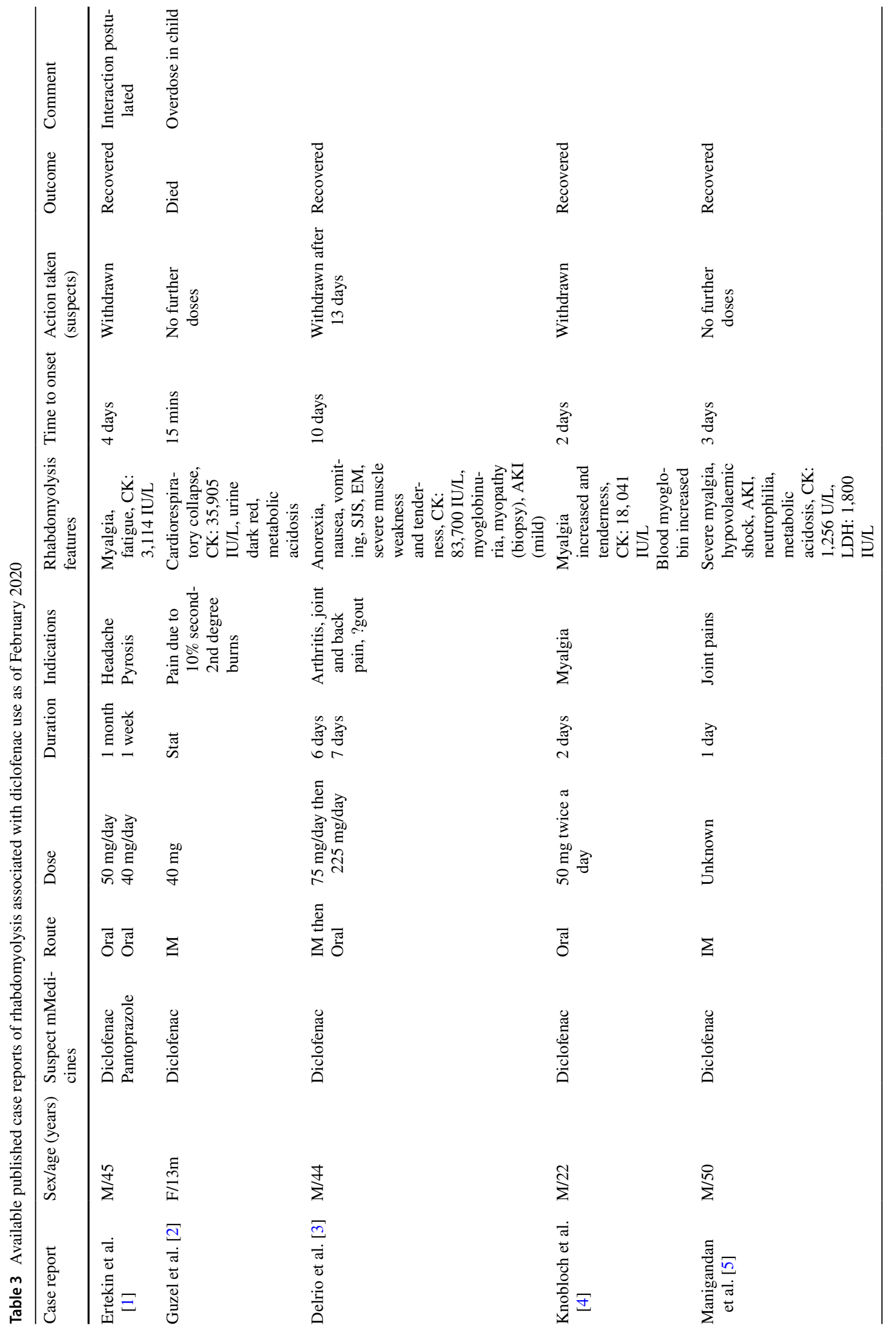




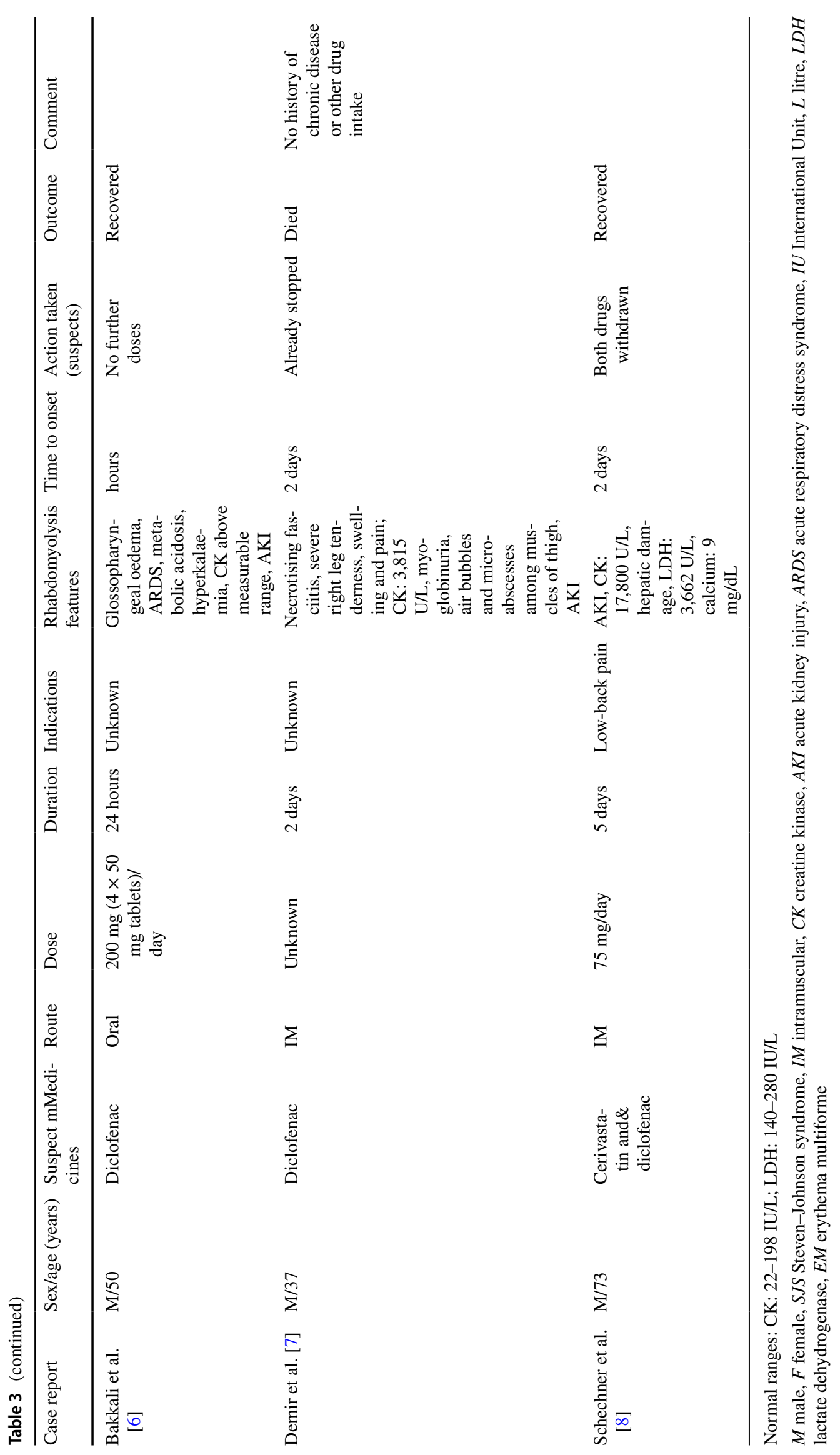


Fig. 1 Summary results of the data mining inn the WHO global database of individual case safety reports (ICSRs) for the association of diclofenac and rhabdomyolysis using the MedDRA System Organ Class (SOC) and Preferred Terms (PTs) and diclofenac as substance (not in combination)

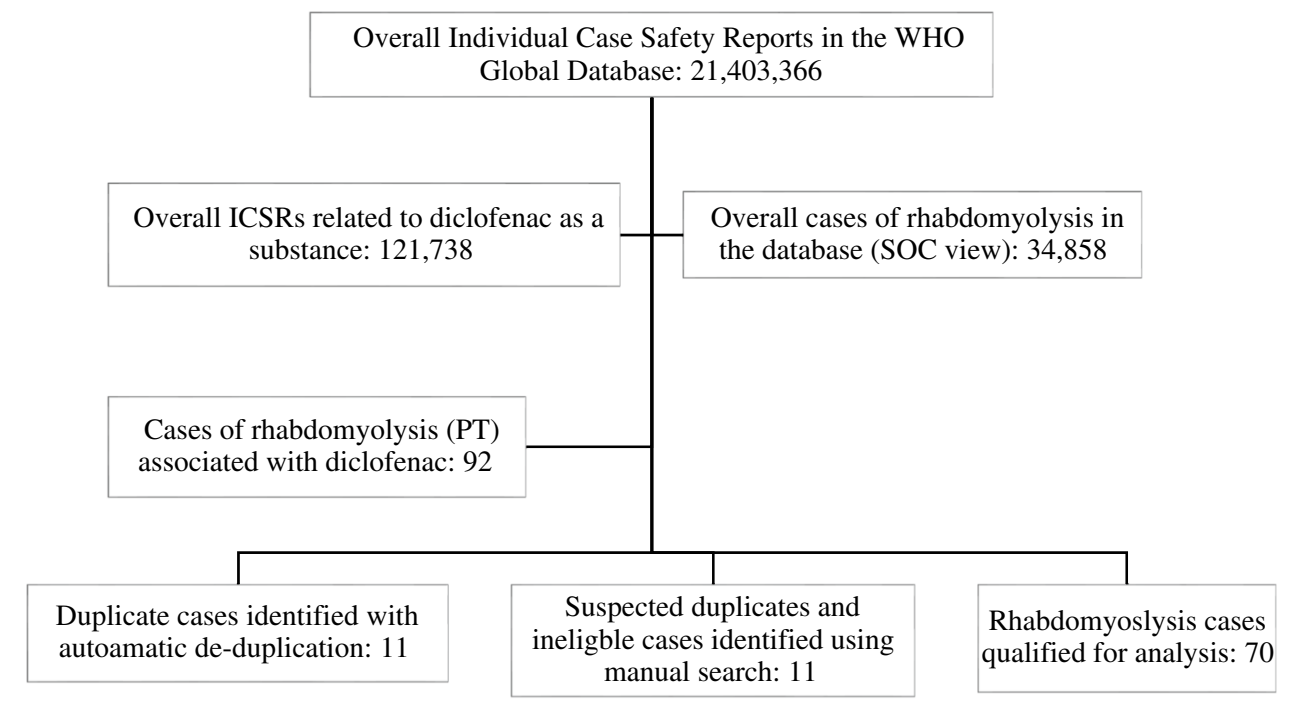

\subsection{Results of Causality Assessment}

In VigiBase, the calculated $\mathrm{IC}_{025}$ value of this drug-reaction combination was below zero (-1.4); indicating that the diclofenac/rhabdomyolysis combination was not statistically prominent from background reporting. However, the close temporal relationship between diclofenac administration and rhabdomyolysis onset in many reports suggested that a causality assessment of the VigiBase case reports should be undertaken. Using the WHO-UMC system for standardized case-causality assessment, the ten reports in which diclofenac was the sole suspect or sole medicine administered and full or partial recovery had occurred after diclofenac withdrawal were scrutinized. Five of the ten reports did not include alternative explanations for rhabdomyolysis such as concomitant medicines and/or co-morbidities and these were classed as 'probable'. Two other reports that did include a co-suspect medicine were also classified as 'probable' for the following reasons. In one, celecoxib was reported as co-suspect but the patient recovered following withdrawal of diclofenac only and no other alternative explanation was found. In the other report, diclofenac and pantoprazole were reported as interacting leading to rhabdomyolysis. However, the interaction theory is not sound and there is no external evidence for proton pump inhibitors causing rhabdomyolysis; hence this report was categorized as 'probable' for diclofenac. Of these seven 'probable' reports in VigiBase, three are shown in Table 3 as they were also published $[1,5,6]$ and the remainder are shown in Table 4.

A further five patients, including the case report from Eritrea, developed rhabdomyolysis subsequent to injection-site tissue necrosis, probably or certainly caused by diclofenac, so that it was an indirect cause of rhabdomyolysis.
No case reports were assessed as showing a 'certain' direct causal relationship as no patients were re-challenged. The majority of the remainder of the reports were assessed as 'possible' as there were co-suspect or concomitant medicines or clinical conditions that could have been alternative explanations for rhabdomyolysis. This group also included patients who appeared to develop rhabdomyolysis indirectly as a result of adverse reactions that were possibly diclofenac related such as purpura fulminans, hepatic necrosis and seizures.

\subsection{Sub-Group Analysis}

\subsubsection{Intramuscular Administration}

Because the patient in our case history was given diclofenac by IM administration and IM diclofenac was given in four fatalities that seemed most likely to be attributable to diclofenac, we analysed separately the 14 reports where diclofenac was given IM. There were seven females and seven males with a median age of 46.0 years (range 1-75 years) in this group. Five described tissue necrosis associated with the diclofenac injection and one purpura fulminans, which can be caused by diclofenac injection or infection. All of these may have caused sufficient tissue damage that could have led to rhabdomyolysis. It is important to highlight that in 11 of the 14 cases administered IM (including the well-documented case presentation reported from Eritrea), diclofenac was the sole suspect and, in nine of these, diclofenac was solely administered. Indications for IM diclofenac, reported for 11 patients, were arthralgia, spondyloarthropathy, aching joints, lumbago, unspecified pain or ache, abdominal pain, depression, acute febrile illness and common cold syndrome (headache, fever and muscle pain). 
Table 4 Unpublished individual case safety reports of rhabdomyolysis associated with diclofenac in the WHO global database with causality classified as " "probable"”

\begin{tabular}{|c|c|c|c|c|c|c|c|c|}
\hline Report number & $\begin{array}{l}\text { Medicines both } \\
\text { suspected (S) } \\
\text { and Concomi- } \\
\text { tant (C) }\end{array}$ & Route & Indication & Time to onset & Reaction & $\begin{array}{l}\text { Action taken } \\
\text { with medicines }\end{array}$ & Outcome & $\begin{array}{l}\text { Notes/ refer- } \\
\text { ences }\end{array}$ \\
\hline 1 & $\begin{array}{l}\text { Diclofenac (S) } \\
\text { Bupivacaine } \\
\text { (C) }\end{array}$ & $\begin{array}{l}\text { Rectal } \\
\text { Epidural }\end{array}$ & $\begin{array}{c}\text { Unknown, } \\
\text { nNormal } \\
\text { delivery }\end{array}$ & 3 days & $\begin{array}{l}\text { Rhabdomy- } \\
\text { olysis }\end{array}$ & $\begin{array}{l}\text { Diclofenac } \\
\text { withdrawn }\end{array}$ & Recovered & \\
\hline 2 & $\begin{array}{l}\text { Diclofenac (S) } \\
\text { Rebamipide (C) }\end{array}$ & $\begin{array}{l}\text { Unknown } \\
\text { Unknown }\end{array}$ & Myalgia & 3 months & $\begin{array}{l}\text { Rhabdomy- } \\
\text { olysis, CK: } \\
490.5 \text { IU/L, } \\
\text { bBlood myo- } \\
\text { globin: } 940\end{array}$ & $\begin{array}{l}\text { Diclofenac } \\
\text { withdrawn } \\
\text { Rebamipide } \\
\text { continued }\end{array}$ & Recovered & \\
\hline 3 & $\begin{array}{l}\text { Diclofenac (S) } \\
\text { Celecoxib (S) } \\
\text { Paracetamol } \\
\text { (C) }\end{array}$ & Unknown & Pain & Unknown & $\begin{array}{l}\text { Rhabdomy- } \\
\text { olysis }\end{array}$ & $\begin{array}{l}\text { Diclofenac and } \\
\text { paracetamol } \\
\text { withdrawn; } \\
\text { celecoxib } \\
\text { continued }\end{array}$ & Recovering & $\begin{array}{l}\text { Very small } \\
\text { amount of } \\
\text { published } \\
\text { case report } \\
\text { evidence } \\
\text { for par- } \\
\text { acetamol } \\
\text { being } \\
\text { causal }\end{array}$ \\
\hline 4 & Diclofenac & Unknown & Backache & $\begin{array}{r}\text { Within } 5 \\
\text { months }\end{array}$ & $\begin{array}{l}\text { Rhabdomy- } \\
\text { olysis } \\
\text { AKI }\end{array}$ & $\begin{array}{l}\text { Diclofenac } \\
\text { withdrawn }\end{array}$ & Recovered & Dehydrated \\
\hline
\end{tabular}

Normal ranges: CK: 22-198 IU/L; LDH: 140-280 IU/L

$I M$ intramuscular, $C K$ creatine kinase, $A K I$ acute kidney injury, $I U$ International Unit, $L$ litre, $L D H$ lactate dehydrogenase

\subsubsection{Reports with Co-Prescribed Lipid-Lowering Agents}

There were 27 reports in which LL agents, known to cause rhabdomyolysis, were listed as co-suspect, interacting or concomitant with diclofenac. These included 17 males and ten females with a median age of 66.0 years (range 41-90). Indications for diclofenac in these reports were joint-related (6), other specified musculoskeletal pain (3), unspecified pain (2), headache (1) and pyrexia (1). For 14 patients the indication was not stated. Routes of diclofenac administration were oral (16), IM (2), cutaneous (2), rectal (1) and not stated (6). At the time of reporting, 17 patients had recovered or were recovering, three had recovered with sequelae and two had not recovered from rhabdomyolysis. The outcome for the remaining five was unknown. There were no reports of fatalities, but for eight patients the rhabdomyolysis was life threatening.

The TTO of rhabdomyolysis was assessed from the start dates of diclofenac and LL agents. The median TTOs for diclofenac, where stated precisely (ten reports), was 4.5 days (range 0-19). Two other reports gave TTOs for diclofenac of 2-3 months and 1-2 years. TTOs for LL agents were stated in ten reports. For nine reports TTOs for LL agents ranged widely from 0 days to 9 years (6 within 4 months and three at 2, 5 and 9 years). In the tenth report, three LL agents were listed and diclofenac was commenced after the onset dates for rhabdomyolysis and acute kidney injury (AKI).
The proportion of patients who developed AKI with rhabdomyolysis in the LL group was $62.9 \%$ (17/27) compared with $37.1 \%$ for all of the 70 reports. Diclofenac alone can cause or exacerbate AKI. Concomitant medicines that may have contributed to AKI include: angiotensin-converting enzyme inhibitors (ACEis) and angiotensin 2 receptor blockers (A2RBs) $(11 / 27,40.7 \%)$. Only three of the remaining 43 patients in the whole dataset of 70 were taking either ACEis or A2RBs (7.0\%). All eight patients who were taking the 'triple whammy' (NSAID, diuretic and ACEi or A2RB), which predisposes to renal failure, were in this LL group and all developed AKI with rhabdomyolysis. Another risk was that four patients were taking additional NSAIDs concomitantly with diclofenac.

\section{Discussion}

The multifactorial nature of rhabdomyolysis makes this causal association challenging. On top of this, several published and VigiBase case reports had potential confounders that would lead to confounding bias. Nevertheless, the short time interval in a substantial number of reports between starting diclofenac and onset of rhabdomyolysis suggests that there is a relationship between the two. Our study suggests four possible explanations for the close temporal 
relationship between diclofenac use and rhabdomyolysis: (1) A small number of published and VigiBase case reports suggest diclofenac may occasionally be directly causal; (2) diclofenac may be indirectly causal following extensive tissue necrosis with IM injection or other diclofenac-related adverse reactions; (3) diclofenac may transform subclinical rhabdomyolysis with LL agents to overt disease and increase the risk of associated AKI; (4) the relationship may be noncausal if the symptoms for which diclofenac was prescribed were due to rhabdomyolysis.

The fact that diclofenac was reported as a sole suspected medicine and solely administered in a substantial number of cases shows specificity of the association and, thus, strengthens causation. To minimize the effect of confounders, causality was assessed using the WHO-UMC criteria. Reports were sought in which diclofenac was reported as a sole suspect and the patients recovered following withdrawal of diclofenac (positive dechallenge) with no reported alternative explanations for rhabdomyolysis. These reports, classified as 'probable', provide the best evidence in VigiBase of a causal relationship between the two. There was also evidence of an indirect causal effect particularly related to injection-site necrosis.

The strength of association or IC value was found to be negative for this combination in VigiBase. This tells us that rhabdomyolysis has not been more frequently reported than expected in the WHO global database, which is not in favour of the causal association. However, disproportionality analysis is a filter that reduces the possibility of missing causal associations but a lack of a statistical signal should not deter further investigation if there are well-documented ICSRs with evidence of causality. The eight published case reports that associate rhabdomyolysis and diclofenac [1-8] and the cases reported in VigiBase, submitted by different countries, indicate that the association is consistent. This observation is also supported by a study that assessed 8,610 cases of drug-induced rhabdomyolysis reported to the FDA, in which 100 cases were associated with diclofenac [24]. This is in excess of the reports from the USA in VigiBase since the FDA database includes reports from market-authorization holders of adverse events occurring in non-USA countries if the medicine is marketed in the USA.

One possible mechanism by which diclofenac could cause rhabdomyolysis has been hypothesized. An in vitro study involving artificially expressing human organic anion transporter (hOAT) receptors showed that hOAT3 interacted with acetaminophen and diclofenac [25]. Expression of hOAT3 is predominantly in the kidneys but it has been demonstrated in skeletal muscle using Northern blot analysis. The authors stated that although precise immunohistochemical analysis should be performed, it is possible that hOAT3 mediates the accumulation of NSAIDs in skeletal muscle. Hypothetically this could lead to the induction of rhabdomyolysis in some individuals, but it requires to be substantiated with further studies [25].

Diclofenac may also indirectly cause rhabdomyolysis as a complication of a known diclofenac-related adverse reaction. For example, the five reports of injection-site reactions causing extensive tissue necrosis, where rhabdomyolysis appears to be a consequence, might be because of muscle injury and infection. One of the published case reports not in VigiBase also describes a similar indirect effect through necrotizing fasciitis that was fatal [7]. The indications in some reports, such as short-term non-specific muscle pain, suggest that another explanation for an observed association is the inadvertent use of diclofenac to treat early unrecognized rhabdomyolysis symptoms leading to confounding by indication.

The study also highlights off-label use of diclofenac and inappropriate IM administration. It is important to reflect that in 11 of the 14 cases administered IM, diclofenac was the sole suspect and in nine cases diclofenac was solely administered. Also, this group was younger on average than the total dataset, and four of the seven fatalities in the whole dataset were related to IM injection (three with injection-site necrosis or purpura fulminans and one due to an overdose in a child). Prevention of this rare but serious injection-site reaction should be possible in many cases. As diclofenac is a commonly prescribed and potentially overused drug, healthcare professionals need to be aware of the potential risk and take some measures to prevent or minimize it. Taking the lowest effective dose for the shortest possible time, administering deep intragluteal injection into the upper outer quadrant, and using the Z-track injection method (believed to be a safe method of IM injection) would prevent or minimize tissue necrosis that triggers rhabdomyolysis [26, 27].

Prevention may also be possible if off-label use, such as for fever and respiratory symptoms, is avoided and IM administration is confined to patients for whom there is a strong indication for this medicine and who cannot take it by the oral or rectal route. According to the EMA/MHRA SmPC, therapeutic indications of diclofenac are rheumatoid arthritis, osteoarthrosis, low back pain, migraine attacks, acute musculoskeletal disorders and trauma, ankylosing spondylitis, acute gout, control of pain and inflammation in orthopaedic, dental and other minor surgery, pyrophosphate arthropathy and associated disorders [9].

AKI was the most frequently co-reported reaction with rhabdomyolysis, at $37.1 \%$, which is within the reported range of $10-50 \%[14,28]$. However, in the subgroup of patients taking LL agents with diclofenac, the proportion of AKI was much higher at 62.9\% (17/27). This is an important observation since development of AKI appears to increase the risk of a fatal outcome with rhabdomyolysis [28]. It is known that rhabdomyolysis may be subclinical [29], and, hence, it is possible that the addition of diclofenac to LL agents aggravated it or increased the risk of associated AKI. It is 
also possible that diclofenac was prescribed for early and unrecognized symptoms of rhabdomyolysis in these patients.

During rhabdomyolysis, water is taken up into muscle tissue leading to extracellular hypovolaemia and therefore vasoconstriction. Diclofenac blocks the renal prostaglandin activity that would allow vasodilatation in the kidney in these circumstances. Hence, glomerular blood flow is reduced and renal impairment can occur. Furthermore, the myoglobin released during rhabdomyolysis is easily filtered into the urine and causes renal tubular obstruction [14, 30-32]. Reabsorption of myoglobin can occur in the proximal renal tubules but any adverse effects of diclofenac on renal tubules could theoretically inhibit this reabsorption. As $40 \%$ of the patients taking LL agents were on ACEis or angiotensin receptor blockers and the majority of these were also taking diuretics, the adverse effect of diclofenac on kidney function would have been compounded [33].

\subsection{Limitations}

This study has some limitations. The cases are captured from the WHO global database of ICSRs, which holds suspected cases that vary with regard to their source and completeness. Thus, the authors could not validate the diagnosis of rhabdomyolysis. Due to lack of denominator information in spontaneous reporting, this study cannot quantify the incidence of rhabdomyolysis associated with diclofenac. The subgroup numbers and numbers of fatalities are small so that larger studies are needed to confirm or refute the hypotheses generated from the observations.

\subsection{Conclusion}

In conclusion, the plausible temporal relationship, specificity and consistency of the association observed, and the suggested possible biological mechanism, are in agreement with several Bradford Hill criteria. In addition, the cases with positive dechallenge and the published and VigiBase cases fulfilling WHO-UMC causality criteria suggest a possible causal link between diclofenac and rhabdomyolysis. It is, however, important to note that this causal inference is made based on the limited available information in the medical literature and WHO global database of ICSRs; thus, conclusions could change in the future with studies that may strengthen or weaken the evidence.

Rhabdomyolysis is a life-threatening condition that could lead to complications such as metabolic instability, acute renal failure, disseminated intravascular coagulation and cardiac arrest [34], and thus avoidance of diclofenac where possible, early diagnosis and aggressive management are important to avoid its complications. From a practical aspect, it is important to reflect on the rare but potentially fatal reactions related to IM diclofenac, the young age of those fatally affected, and the increased risk of serious renal complications of rhabdomyolysis attributed to the concomitant use of LL agents, diclofenac and other agents that may contribute. As diclofenac is among the commonly prescribed and potentially overused drugs [35], avoidance of off-label use and unnecessary IM administration, especially for minor conditions, and correct administration where it is indicated are likely to reduce the risk. Also, before diclofenac is prescribed, there needs to be alertness to the possibility that unexplained musculoskeletal symptoms may be early manifestations of rhabdomyolysis. It is also well known that combinations of diclofenac with other agents that synergistically adversely affect renal function should be avoided. Finally, we recommend healthcare professionals be aware that diclofenac needs to be discontinued if rhabdomyolysis is suspected.

Acknowledgements We would like to thank Dr. Qun-Ying Yue from the Uppsala Monitoring Centre, Sweden, for her review and valuable contributions.

\section{Declarations}

Funding No funding was used for this study.

Conflict of Interest The authors declare that they have no competing interests.

Ethics Approval Information of all cases retrieved from the WHO global database of individual case safety reports is de-identified and ethical approval is not required.

Consent to Participate Informed consent to participate in the study was obtained from the Eritrean patient (case report).

Consent for Publication Though informed consent was obtained from the case reported from Eritrea, the patient's details (including name, images, residence, admitting and referral hospitals) are also completely anonymized.

Availability of Data and Material The datasets generated and analyzed during the current study are not publicly available due to agreements between contributors of data to the database used (VigiBase) and the custodian of the database. National Centres (mainly drug regulatory authorities) constituting the WHO Programme for International Drug Monitoring (PIDM) contribute data to VigiBase and the Uppsala Monitoring Centre is the custodian in its capacity as WHO Collaborating Centre for International Drug Monitoring. Some subsets of the data may be available from the corresponding author on reasonable request.

Code Availability Not applicable.

Author Contributions MR and YF contributed to the conception the study. The case from Eritrea was reported, investigated and written by AA and all the authors contributed to the study design. RS, MR and YF contributed to the analysis of publications, data analysis and their interpretation. MR and RS drafted the article, and it was reviewed by the rest of the authors. Finally, all authors read and approved the final manuscript. 
Disclaimer The authors are indebted to the National Centres that make up the WHO Programme for International Drug Monitoring and contribute reports to VigiBase. However, the opinions and conclusions of this study are not necessarily those of the various centres nor of the WHO.

Open Access This article is licensed under a Creative Commons Attribution-NonCommercial 4.0 International License, which permits any non-commercial use, sharing, adaptation, distribution and reproduction in any medium or format, as long as you give appropriate credit to the original author(s) and the source, provide a link to the Creative Commons licence, and indicate if changes were made. The images or other third party material in this article are included in the article's Creative Commons licence, unless indicated otherwise in a credit line to the material. If material is not included in the article's Creative Commons licence and your intended use is not permitted by statutory regulation or exceeds the permitted use, you will need to obtain permission directly from the copyright holder. To view a copy of this licence, visit http://creativecommons.org/ licenses/by-nc/4.0/.

\section{References}

1. Ertekin YH, Yakar B, Ertekin H, et al. Diclofenac- and pantoprazole-induced Rhabdomyolysis: a potential drug interaction. Drug Saf Case Rep. 2015;10:1-4. https://doi.org/10.1007/ s40800-015-0012-6.

2. Güzel A, Biner Orhaner B, Aylanç H. Fatal acute diclofenacinduced Rhabdomyolysis in a pediatric patient. Balkan Med J. 2011;28:102-3. https://doi.org/10.5174/tutfd.2009.02192.0.

3. Delrio FG, Park Y, Herzlich B, Grob D. Diclofenac-lnduced rhabdomyolysis. Am J Med Sci. 1996;312:95-7. https://doi.org/10. 1016/S0002-9629(15)41764-1.

4. Knobloch K, Rossner D, Gössling T, Lichtenberg A, Richter M, Krettek C. Rhabdomyolysis after administration of diclofenac. Unfallchirurg. 2005;108(5):415-7. https://doi.org/10.1007/ s00113-004-0874-z.

5. Manigandan G, Seshadri MS. Diclofenac-induced rhabdomyolysis—a great masquerader. J Assoc Phys India. 2016;64:90-1.

6. Bakkali H, Belyamani L, Massou S, Elwartiti L, Aboulaala $\mathrm{K}$, Balkhi H, Haimeur C. Rhabdomyolysis associated to glossopharyngeal edema: a rare side effect of diclofenac. Am J Clin Exp Med. 2014;2:161-4. https://doi.org/10.11648/j.ajcem.20140 206.18.

7. Demir BF, Katipoglu B, Yirgin G, Ates I. A rare case due to intramuscular diclofenac injection: necrotizing fasciitis, Rhabdomyolysis and acute kidney injury. Ulutas Med J. 2018;4:50-52. https://www.bibliomed.org/?mno=290455.

8. Schechner V, Hershcovici T, Beigel Y. Rhabdomyolysis due to the combined therapy with Cerivastatin and diclofenac. J Pharm Technol. 2003;19:219-21.

9. UK. Electronic Medicines Compendium (emc). Summary of product characteristics of diclofenac tablet50mg. 2020. https://www. medicines.org.uk/emc/product/4333/smpc. Accessed 4 May 2020.

10. US FDA. Prescribing information of diclofenac sodium delayed release tablet. 2020. https://www.drugs.com/pro/diclofenac.html. Accessed 4 May 2020.

11. European Medicines Agency. Assessment report for diclofenac containing medicinal products (systemic formulations). EMA/544760/2013. September 2013. 2013. https://www.ema. europa.eu/documents/referral/diclofenac-article-31-referral-pracassessment-report_en.pdf.
12. Australian Government, Department of Health. Therapeutic Goods Administration. Safety review of diclofenac. Version 2.1, October 2014. 2014.

13. Keltz E, Khan FY, Mann G. Rhabdomyolysis. The role of diagnostic and prognostic factors. Muscles Ligaments Tendons J. 2014;3:303-12. https://www.ncbi.nlm.gov/pmc/articles/PMC39 40504/.

14. Petejova N, Martinek A. Acute kidney injury due to rhabdomyolysis and renal replacement therapy: a critical review. Crit Care. 2014;18:224. http://ccforum.com/content/18/3/224.

15. Lindquist M. VigiBase, the WHO Global ICSR Database System: basic facts. Drug Inf J. 2008;42:409-19.

16. https://www.meddra.org/. Accessed Feb 2020.

17. Bate A, Lindquist M, Edwards IR, Olsson S, Orre R, Lansner A, et al. A Bayesian neural network method for adverse drug reaction signal generation. Eur J Clin Pharmacol. 1998;54:315-21.

18. WHO-Uppsala Monitoring Centre. The use of the WHO-UMC system for standardized case causality assessment. 2020. https:// www.who-umc.org/media/164200/who-umc-causality-asses sment_new-logo.pdf. Accessed 4 May 2020.

19. Shakir SA, Layton D. Causal association in pharmacovigilance and pharmacoepidemiology: thoughts on the application of the Austin Bradford-Hill criteria. Drug Saf. 2002;25(6):467-71.

20. Buckingham R (ed). Martindale: The Complete Drug Reference. [online] London: Pharmaceutical Press. http://www.medicinesc omplete.com.cmezproxy.chmeds.ac.nz/. Accessed on 20 May 2020.

21. www.micromedexsolutions.com. Accessed on 20 May 2020.

22. Kuhn M, Letunic I, Jensen LJ, Bork P. The SIDER database of drugs and side effects. Nucl Acids Res. 2015. https://doi.org/ 10.1093/nar/gkv1075. http://sideeffects.embl.de/drugs/3032/. Accessed on 13 Jan 2021.

23. Novartis Pharmaceuticals UK Ltd. Summary of product characteristics of diclofenac sodium (Voltarol ampoules) $75 \mathrm{mg} / 3 \mathrm{ml}$ ). Updated January 2020. 2020. https://www.medicines.org.uk/emc/ product/1043/smpc https://www.medicines.org.uk/emc/product/ 4333/smpc. Accessed 4 May 2020.

24. Oshima Y. Characteristics of drug-associated rhabdomyolysis: analysis of 8,610 cases reported to the U.S. Food and Drug Administration. Intern Med. 2011;50:845-53. https://doi.org/10. 2169/internalmedicine.50.4484.

25. Khamdang S. Interactions of human organic ion transporters and human organic cation transporters with nonsteroidal anti-inflammatory drugs. Pharmacology. 2002;303:534-9.

26. Uri O, Behrbalk E. Tissue necrosis following intramuscular administration of various drugs (Nicolau syndrome): Clinical presentation, pathophysiology and treatment. Harefuah. 2009;148:186-8.

27. Giving Z-track injections, Nursing. 2002;32(9):81. https://journ als.1ww.com/nursing/fulltext/2002/09000/giving_z_track_injec tions.56.aspx.

28. Ward MM. Factors predictive of acute renal failure in rhabdomyolysis. Arch Intern Med. 1988;148:1553-7.

29. Huerta-Alardin AL, Varon J, Marik PE. Bench-to-bedside review: rhabdomyolysis: an overview for clinicians. Crit Care. 2005;9:1158-69. https://doi.org/10.1186/cc2978.

30. Daher E, Zanetta DM, Cavalcante MB, et al. Risk factors for death and changing patterns in leptospirosis acute renal failure. Am J Trop Med Hyg. 1999;61:630.

31. Lima RS, da Silva Junior GB, Liborio AB, et al. Acute kidney injury due to rhabdomyolysis. Saudi J Kidney Dis Transpl. 2008;19:721.

32. Daher Ede F, Zanetta DM, Abdulkader RC. Pattern of renal function recovery after leptospirosis acute renal failure. Nephron Clin Pract. 2004;98:c8-14. 
33. Toto RD. Renal insufficiency due to angiotensin-converting enzyme inhibitors. Miner Electrol Metab. 1994;20:193-200.

34. Counselman FL. Rhabdomyolysis. Emergency Medicine Comprehensive study guide, 5th edn., vol. 271. McGraw-Hill Companies; 2000. pp. 1841-5.
35. Internet: China Diclofenac Investigation Market Report, 20192023. 2020. https://www.businesswire.com/news/home/20190 $822005517 /$ en/China-Diclofenac-Investigation-Market-Report2019-2023. Accessed on 13 Jan 2021. 